\title{
LIGO optics: initial and advanced
}

Jordan Camp, Garilynn Billingsley, William P. Kells, Albert Lazzarini, Gary H. Sanders, et al.

Jordan Camp, GariLynn Billingsley, William P. Kells, Albert Lazzarini, Gary H. Sanders, Stanley L. Whitcomb, A. Alexandrovski, Martin M. Fejer, Eric K. Gustafson, Roger K. Route, Sheila Rowan, B Bochner, Gregory M. Harry, Nergis Mavalvala, Rainer Weiss, James Hough, "LIGO optics: initial and advanced," Proc. SPIE 4679, Laser-Induced Damage in Optical Materials: 2001, (9 April 2002); doi: 10.1117/12.461689

SPIE. Event: Boulder Damage, 2001, Boulder, CO, United States 


\title{
LIGO optics: initial and advanced
}

\author{
J.B. Camp, G. Billingsley, W. Kells, A. Lazzarini, G. Sanders, S. Whitcomb \\ LIGO Project, California Institute of Technology, Pasadena, Ca. 91125
}

A. Alexandrovski, M. Fejer, E. Gustafson, R. Route, S. Rowan

Ginzton Laboratory, Stanford University, Stanford, Ca. 94305

B. Bochner, G. Harry, N. Mavalvala, R. Weiss

LIGO Project, Massachusetts Institute of Technology, Cambridge, Mass. 02138

\author{
J. Hough \\ Dept. of Physics and Astronomy, University of Glasgow, G128QQ, Scotland
}

\begin{abstract}
The LIGO project has completed the installation of large fused silica optical components in the vacuum systems of its observatories. Commissioning work on the Hanford $2 \mathrm{~km}$ interferometer has determined an upper limit to the optics losses, allowing comparison with design and pre-installation testing. Planning and development of sapphire optics for the next generation, advanced LIGO detector is now underway, including polishability, optical homogeneity, absorption, and birefringence. The advanced optics development also includes research aimed at lowering coating loss.
\end{abstract}

Keywords: LIGO optics, substrates, coatings, sapphire, absorption, birefringence

\section{INTRODUCTION}

The search for astrophysical sources of gravitational radiation in the next decade will involve the construction of long baseline laser interferometers by a number of research groups. One of these, the LIGO project ${ }^{1}$, has the configuration of a Michelson interferometer with $4 \mathrm{~km}$ length Fabry-Perot arms. The optical components which comprise the LIGO I interferometer are the recycling mirror, beam splitter, and two input test masses and two end test masses (see fig. 1).

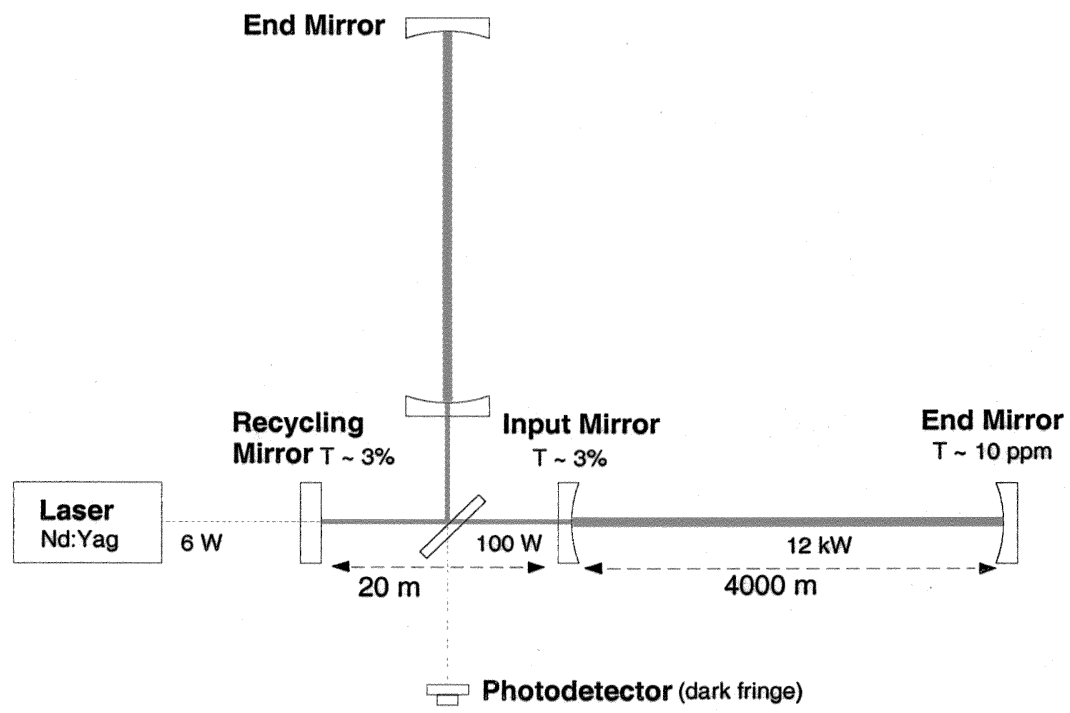

Figure 1: Schematic diagram of LIGO interferometer 
The performance of the optical components must be consistent with the specified LIGO shot noise limited sensitivity, which translate into requirements on the total stored power in the LIGO resonant cavities, and the power at the interferometer dark port. These requirements in turn drive specifications for the following optical parameters: size, radius of curvature, transmission, surface figure, scatter loss, absorption loss, and optical homogeneity.

The optics of the LIGO I interferometers have been installed in the vacuum systems of the Hanford and Livingston sites, and commissioning of these interferometers is now underway. At the same time, research and development of the next generation, advanced LIGO detector is proceeding. The design sensitivity of both the initial and advanced interferometers is shown in figure 2, where the strain $h$ equals $\Delta \mathrm{L} / \mathrm{L}$, with $\mathrm{L}$ the detector length and $\Delta \mathrm{L}$ the displacement sensitivity. The advanced sensitivity is shown for both fused silica and sapphire optics.

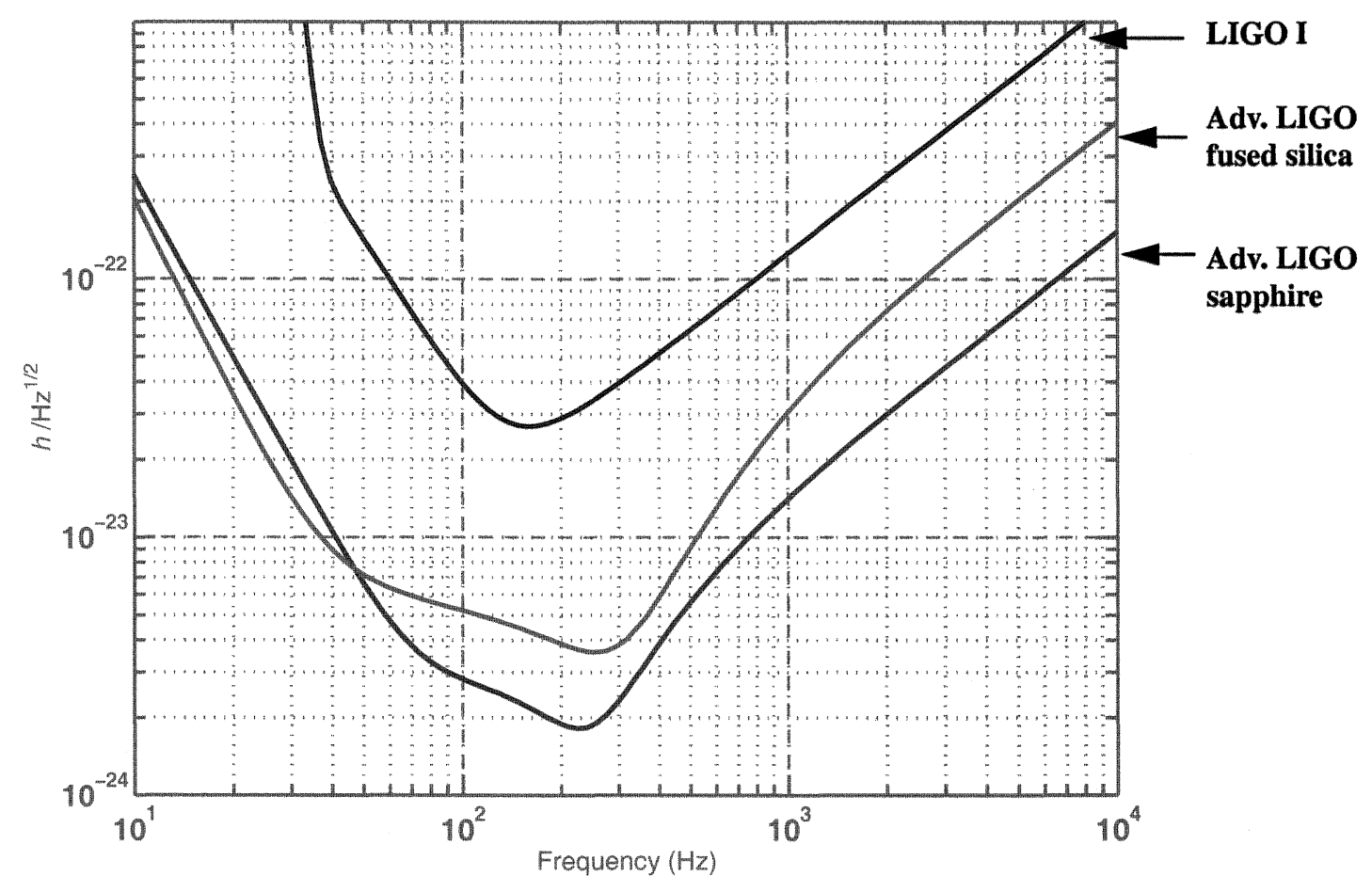

Figure 2: Interferometer sensitivity for LIGO I and advanced LIGO detectors

The advanced interferometer uses active seismic isolation, multiple pendulum suspensions, and a factor of 60 higher stored laser power relative to the initial detector to achieve the increased sensitivity. The current planning is to use sapphire optics for this detector, because of its superior thermal noise performance and its higher thermal conductivity.

In this paper we summarize the status of the LIGO optics, including the initial optics, presently installed and under commissioning, and the advanced detector optics, under development. Section 2 lists the optics requirements for LIGO I, and describes the measurement devices developed at Caltech, including a $1064 \mathrm{~nm}$ phase measuring interferometer for surface figure characterization, and a scatterometer to measure optical scatter loss. Characterization of the Hanford $2 \mathrm{~km}$ interferometer has determined an upper limit to the optics losses and has allowed comparison to the design requirements, discussed in section 3. Finally, design and research on the optics for the advanced LIGO interferometer is now underway. Sapphire is the planned optical material for this interferometer, designed for operation at 60 times the power, and 10 times the sensitivity of LIGO I. We discuss in section 4 the research and preliminary results in the sapphire development. 


\section{LIGO I OPTICS REQUIREMENTS AND TESTING}

After extensive evaluation of the state-of-the-art capabilities of the optics industry, the LIGO I optical requirements were derived with the use of an FFT-based optical propagation $\operatorname{code}^{2}$. The code simulates the interferometer performance with the use of surface phase maps for all optics, and includes the effects of optical losses and inhomogeneities. It also includes the effect of thermal distortion from substrate and coating absorption.

The optical requirements were used to derive the specifications summarized in Table I.

Table 1: LIGO I Optics Specifications

\begin{tabular}{|c|c|c|c|c|}
\hline \multirow[t]{2}{*}{ Physical Quantity } & \multicolumn{2}{|c|}{ Test Mass } & \multirow{2}{*}{$\begin{array}{l}\text { Beam } \\
\text { Splitter }\end{array}$} & \multirow{2}{*}{$\begin{array}{l}\text { Recycling } \\
\text { Mirror }\end{array}$} \\
\hline & End & Input & & \\
\hline Diameter $(\mathrm{cm})$ & 25 & 25 & 25 & 25 \\
\hline Thickness (cm) & 10 & 10 & 4 & 10 \\
\hline Radius of Curvature (km) & $7.4+/-1$ & $14.2+/-1$ & $>-720,<200$ & $14.9+/-1$ \\
\hline ROC Matching Tolerance $^{\mathrm{a}}(\mathrm{km})$ & $+/-0.3$ & -1 & - & +0.75 \\
\hline Transmission & $10 \mathrm{ppm}$ & 0.03 & 0.5 & 0.03 \\
\hline Surface Figure $\mathrm{rms}^{\mathrm{b}}(\mathrm{nm})$ & 0.8 & 0.8 & 1.6 & 1.6 \\
\hline Scatter Loss (ppm) & 10 & 10 & 50 & 50 \\
\hline Coating Absorption Loss (ppm) & 1 & 1 & 1 & 1 \\
\hline Substrate Absorption Loss ( $\mathrm{ppm} / \mathrm{cm}$ ) & 50 & 5 & 5 & 15 \\
\hline
\end{tabular}

a. ITM's matched to each other and RM, ETM's matched to each other

b. central $80 \mathrm{~mm}$ diameter; power, astigmatism removed

Briefly, some elaboration of the requirements is as follows. The optics diameter is set large enough to allow the $4 \mathrm{~cm}$ beam spot to suffer less than $1 \mathrm{ppm}$ diffraction loss. The specified radius of curvature and matching tolerance ensures maximum stability of the large beam spot in the $10 \mathrm{~m}$ separation between recycling mirror and input test mass. The surface figure and scatter loss requirements limit the arm cavity loss to order $100 \mathrm{ppm}$ per optic so that a recycling cavity power buildup, defined as the ratio of the power at the beamsplitter to the power incident on the recycling mirror, is at least 30; this satisfies the LIGO I shot noise requirement. Finally, the coating and substrate loss specifications limit the thermal distortion of the input test masses so that the transmitted wavefront distortion does not become unacceptably large $e^{3,4}$.

Testing of the LIGO optics prior to installation was performed to measure the surface profile and surface scatter, to ensure that the optical losses met requirements. The instrumentation has been described in detail in an earlier publication $^{5}$. A summary of the testing follows. 


\subsection{Optical Metrology Interferometry}

A Fizeau type interferometer was used to examine the surface characteristics of the coated LIGO I optics prior to installation. The interferometer was specified for $1064 \mathrm{~nm}$ wavelength so that variations in the coating thickness as well as the surface polish could be determined. The interferometer allows measurement of the surface uniformity by illuminating an optical cavity consisting of a known reference surface and the optic under test with a collimated beam $15 \mathrm{~cm}$ in diameter. Calibration of three different reference surfaces was done using a standard technique of three cavity measurements of the surfaces.

Shown in fig. 3 is a power spectrum of the coated and uncoated Hanford $2 \mathrm{~km}$ recycling mirror surface profile variations, and phase map of the coated optic. The coated optic was measured at Caltech, and the uncoated was measured at the Commonwealth Scientific and Industrial Research Organization (CSIRO) ${ }^{6}$. A best-fit zernike approximation to the phase map was used to remove power and astigmatism before generating the spectra. The sharp spikes in the data are instrumental in origin.
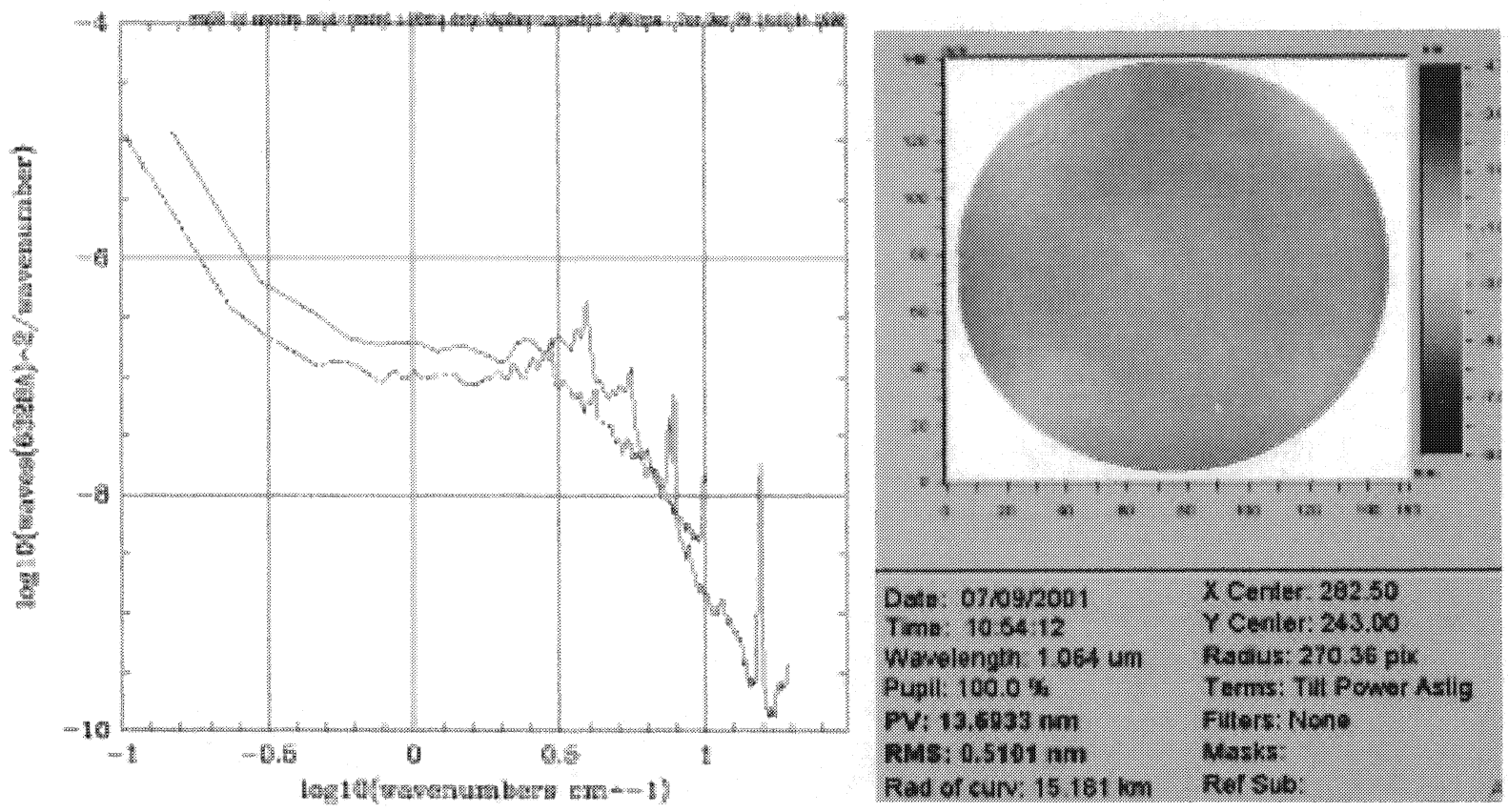

Figure 3: Power spectra and phase map of surface variations of recycling mirror. The upper (lower) curve of the spectra is the coated (uncoated) mirror. The phase map is from the coated optic.

The power spectra are seen to be consistent to within a factor of sqrt(3) in amplitude; the difference is most likely due to an imperfect calibration. With the subtraction of the long range coating-induced curvature by removal of the zernike power term, no evidence is seen for an effect of the coating on the surface variation.

Table 2 shows a set of measurements of the radius of curvature (ROC) of a number of optics, measured by CSIRO before coating and by Caltech after coating. The difference in the ROC in rows 1-3 in Table 2 is attributed to the residual non-uniformity of the coating process, about $5 \mathrm{~nm}$ peak to valley $(\mathrm{P}-\mathrm{V})$ for these $3 \mu \mathrm{m}$ thick coatings. The much larger 
curvature for the beam splitter is believed to be due to residual compressive coating strain, which generates curvature as $(1 / \text { thickness })^{4}$ and has a relatively large effect for this $4 \mathrm{~cm}$ thick optic.

Table 2: Measured ROC of Optics

\begin{tabular}{|c|c|c|c|}
\hline \multirow{2}{*}{ Optic } & \multicolumn{2}{|c|}{ Radius of Curvature } & \multirow{2}{*}{$\begin{array}{c}\text { Coating Induced } \\
\text { Curvature } \\
(\mathrm{nm} \text { P-V) }\end{array}$} \\
\cline { 2 - 3 } & Uncoated $(\mathrm{km})$ & Coated $(\mathrm{km})$ & 7 \\
\hline \hline RM01 & 15.1 & 15.7 & 3 \\
\hline ITM03 & 13.8 & 14.0 & 5 \\
\hline ITM04 & 13.3 & 13.6 & 20 \\
\hline BS01 & -500 & -120 & 7 \\
\hline
\end{tabular}

The surface profile of all the installed optics has been measured. We have found the surface figure rms and P-V, and radius of curvature to meet specifications for all optics, with the exception of the beam splitter. The beam splitter curvature will result in a slight degradation of the recycling cavity stability, causing a decrease in optimal sideband power recycling gain of $\sim 20 \%$.

\subsection{Scatterometer measurements}

Large angle scatter from the surface of a coated core optic has two components. The first is the high spatial frequency variation, or rms microroughness $\left(\sigma_{\mathrm{rms}}\right)$ of the underlying substrate surface. The second is the presence of localized coating defects, typically $1-10 \mu \mathrm{m}$ in size, which may be due to impurities in the coating materials, or a defect or inclusion in the substrate which disrupts the uniformity of the coating process. To compare the two sources of scatter and monitor the optics for coating uniformity, we have used a scatterometer to map the optics surface scatter. All LIGO optics were coated by Research Electro-Optics, Boulder, Co.

The scatterometer directs $80 \mathrm{~mW}$ of light from a $1.06 \mu \mathrm{m}$ wavelength laser, chopped at $\sim 1 \mathrm{kHz}$, normally incident to the optic under test with a $0.8 \mathrm{~mm}$ diameter beam size. Six scatter detectors view the beam spot at 45 degree (135 degree backscatter) through focusing lenses subtending a total solid angle of $\sim 10 \%$. The summed detector output is measured with a lock-in amplifier demodulated at the chopped frequency. The test optic is mounted on a translation stage which automatically scans the optic under the light in two dimensions in steps of $0.4 \mathrm{~mm}$. The measurements were done in a HEPA-filtered environment to minimize dust accumulation. The scatterometer was calibrated by correlating the scatter loss signal with independent ringdown loss measurements at a number of lossy points on an optic. For a given scatter signal, the defect size was obtained by using the calibration to relate the scatter signal to the surface loss, and assuming that the loss was equal to the square of the ratio of the defect size to the beam spot size. The scatter signals were much larger than expected from plus-micron sized aperture diffraction at 45 degree; the scatter was presumably enhanced because of underlying defect structure, such as substrate inclusions or dust trapped in the coating.

Figure 4 shows a scatter histogram of an ETM taken over a $7 \mathrm{~cm} \times 7 \mathrm{~cm}$ area. The average value of the surface microroughness $\sigma_{\text {rms }}$ was provided by the polisher. The broad, non-gaussian peak is due to the variation of substrate microroughness over the surface. The presence of a non-Gaussian tail, due to the presence of coating defects is seen at signals above $\sim 0.6 \mathrm{mV}$; the defect size at this signal level is $\sim 4 \mu \mathrm{m}$. 


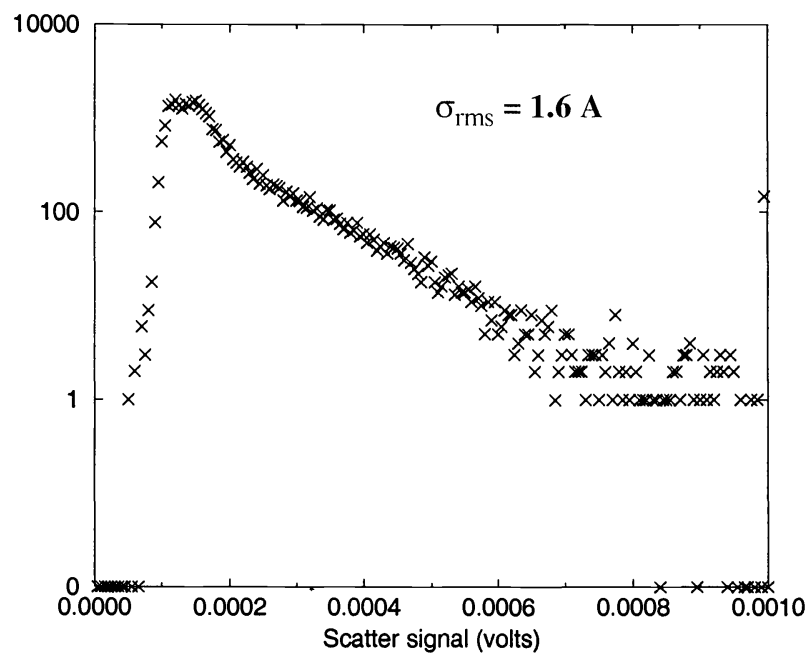

Figure 4: Scatter data from a coated ETM

We find a defect loss for this optic $\sim 1 \mathrm{ppm}$, and a total scatter loss of $8 \mathrm{ppm}$. Uncertainty in the calibration places the errors for the scatter loss at the $50 \%$ level. Measurements of all the installed test masses have shown the optic scatter to be within the $10 \mathrm{ppm}$ loss specifications. The largest defect found for the optics scanned was $\sim 500 \mu \mathrm{m}$ size, and was close enough to the center to cause rejection of the optic. Its appearance suggested a coating flake that settled onto the surface during the coating run.

\section{INTERFEROMETER CHARACTERIZATION OF OPTICS}

At the present time installation of the optics in all three LIGO interferometers has been completed. The commissioning of the Hanford $2 \mathrm{~km}$ interferometer has advanced to the point of locking the full recycled configuration, allowing the measurement of power buildup in the arm cavities. This in turn allows extraction of the system optical losses.

The optical losses of mirrors forming the arm cavity Fabry-Perot may be inferred from the optical gain, ie, the power buildup in the arm. Defining $\mathrm{r}_{\mathrm{arm}}$ as the ratio of the field amplitude reflected from the cavity in resonance $\left(E_{r e f}\right)$ to the incident amplitude $\left(E_{\text {inc }}\right)$, we have:

$$
r_{\text {arm }}=\frac{E_{r e f l}}{E_{\text {inc }}}=r_{1}-\frac{t_{1}^{2} r_{2}}{1-r_{1} r_{2}}
$$

In the above expression, $r_{l}$ and $r_{2}$ are the input and end test mass amplitude reflectivities, and $t_{l}$ is the input test mass transmission. This allows us to calculate $G_{r c}$, which is a measure of the power buildup in the recycling cavity:

$$
G_{r c}=\frac{P_{b s}}{P_{\text {inc }}}=\frac{1}{1+r_{r} \cdot r_{\text {arm }}}
$$


where $r_{r}$ is the recycling mirror reflectivity and $P_{i n c}$ and $P_{b s}$ are the incident power and resonantly enhanced beamsplitter power, respectively. Through use of the expression $r=\sqrt{1-T-L}$ where $T, L$ are the mirror power transmission and power loss, we can relate $G_{r c}$ to the arm cavity optic losses.

Figure 5 shows the power transmitted through the arm cavity for the resonant states of the interferometer as it proceeds from all its cavities unlocked to its fully locked configuration. The highest power state, at the beginning and end of the plot, has the interferometer fully resonant. The next lower power state has the arm cavity locked but the recycling cavity unlocked. The transmitted power ratio of these two states is $G_{r c} d T_{r}$ where $T_{r}$ is the recycling mirror power transmission. The figure shows a measured power ratio of 600 ; the observed values, dependent on interferometer alignment, have been in the range of 600-900. Using the maximum power ratio of 900 and a recycling mirror transmission of 0.028 , we find a recycling factor of 25 , fairly close to the specified value of 30 mentioned in section 2 . From eqns. 1 and 2 , this power buildup is consistent with arm cavity loss per optic $<150 \mathrm{ppm}$. This number is also supported by measurements of the arm cavity reflectivity (eqn. 1). This optic loss upper bound is expected to decrease as further commissioning work is done .

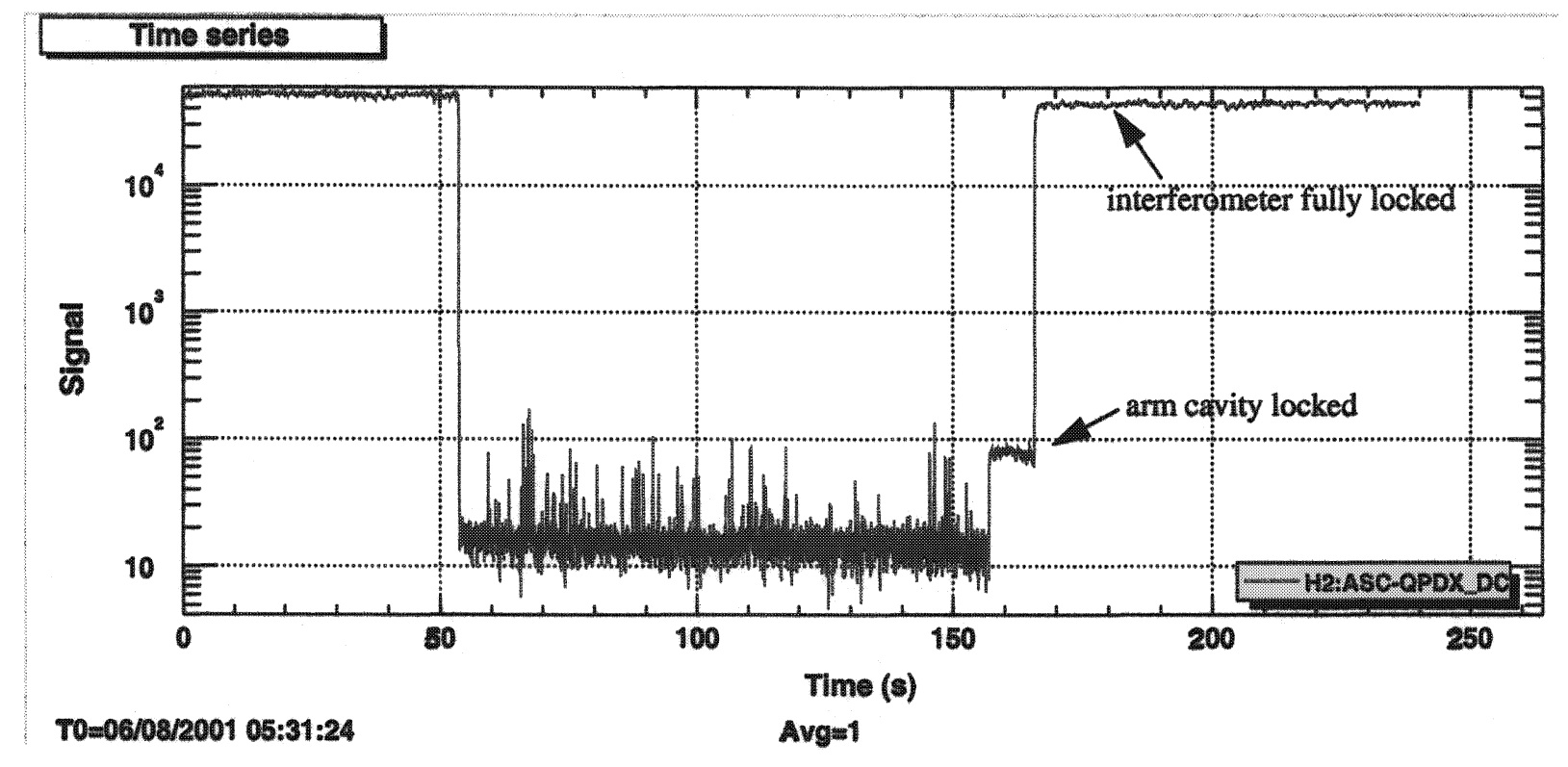

Figure 5: Power buildup in the interferometer 


\section{DEVELOPMENT OF ADVANCED OPTICS}

The advanced LIGO sensitivity curves for fused silica and sapphire are shown in figure 2. Both curves are limited by the optics internal thermal noise in the critical frequency range of 50-300 Hz. In the case of fused silica, the thermal noise is set by the intrinsic mechanical loss of the material well below the resonance frequencies. In the case of the higher Q sapphire, the thermal noise is caused by thermoelastic damping, a coupling of thermal fluctuations in the substrate to surface elastic deformation. The respective curves of figure 2 give an interferometer with sapphire optics a detection range for neutron star binary inspirals of $200 \mathrm{Mpc}$, compared to $140 \mathrm{Mpc}$ for fused silica. Given the cubic dependence of rate on interferometer range, sapphire is seen to offer a significant advantage for the advanced detection rate.

Table 3: Advanced LIGO Sapphire Optics Specifications

\begin{tabular}{|c|c|c|}
\hline \multirow{2}{*}{ Physical Quantity } & \multicolumn{2}{|c|}{ Test Mass } \\
\cline { 2 - 3 } & End & Input \\
\hline Diameter $(\mathrm{cm})$ & 32 & 32 \\
\hline Thickness $(\mathrm{cm})$ & 13 & 13 \\
\hline Radius of Curvature (km) & $50+/-5$ & $50+/-5$ \\
\hline Transmission $^{*}$ & $10 \mathrm{ppm}$ & 0.005 \\
\hline Surface Figure rms $^{\mathrm{a}}(\mathrm{nm})$ & 0.8 & 0.8 \\
\hline Scatter Loss $(\mathrm{ppm})$ & 10 & 10 \\
\hline Coating Absorption Loss $(\mathrm{ppm})$ & 0.1 & 0.1 \\
\hline Substrate Absorption Loss $(\mathrm{ppm} / \mathrm{cm})$ & 100 & 10 \\
\hline Optical Homogeneity $\mathrm{rms}$ & $100 \mathrm{~nm}$ & $10 \mathrm{~nm}$ \\
\hline
\end{tabular}

a. central $120 \mathrm{~mm}$ diameter; power, astigmatism removed

The advanced detector will also operate at an arm cavity stored power 60 times higher than the LIGO I detector. Thermal distortion in fused silica will be very large (section 4.2.2). However, sapphire offers a thermal conductivity 30 times higher than fused silica, potentially limiting thermal distortion to a tolerable level. For these reasons we have undertaken a program to develop sapphire as an optic material.

Table 3 lists the specifications for the advanced sapphire optics. A number of differences with the initial LIGO optics are: longer radii of curvature, yielding a larger beam spot to average over the temperature fluctuations, thus minimizing the thermal noise; larger size to limit edge losses and radiation pressure noise; lower coating absorption to control thermal distortion in the arm cavities; and a requirement on the maximum optical inhomogeneity suffered in transmission through the substrate, to limit wavefront distortion. 


\subsection{Sapphire development}

The sapphire used in the following development studies was provided by Crystal Systems, Inc., Salem, Mass.

\subsubsection{Surface polish and optical homogeneity}

A $15 \mathrm{~cm}$ diameter sapphire test sample has been polished by CSIRO and has been found to satisfy the specifications of Table 3 in both surface figure and scatter loss. Although sapphire is a harder material than fused silica and has a lower removal rate, the polishing capability exists to fabricate optics meeting the surface figure requirements.

A problem in the production of sapphire crystals is distortion of the crystal lattice during the growth phase, resulting in inhomogeneity in the local index of refraction. ${ }^{7}$ Figure 6 shows a phase map taken in transmission through a $15 \mathrm{~cm}$ diameter, $8 \mathrm{~cm}$ thick sapphire crystal grown in the m-axis direction. Long range variations of the order of $100 \mathrm{~nm}$ peak to valley can be observed, as well as short range striations of $10 \mathrm{~nm}$ over a $\mathrm{mm}$ spatial scale.

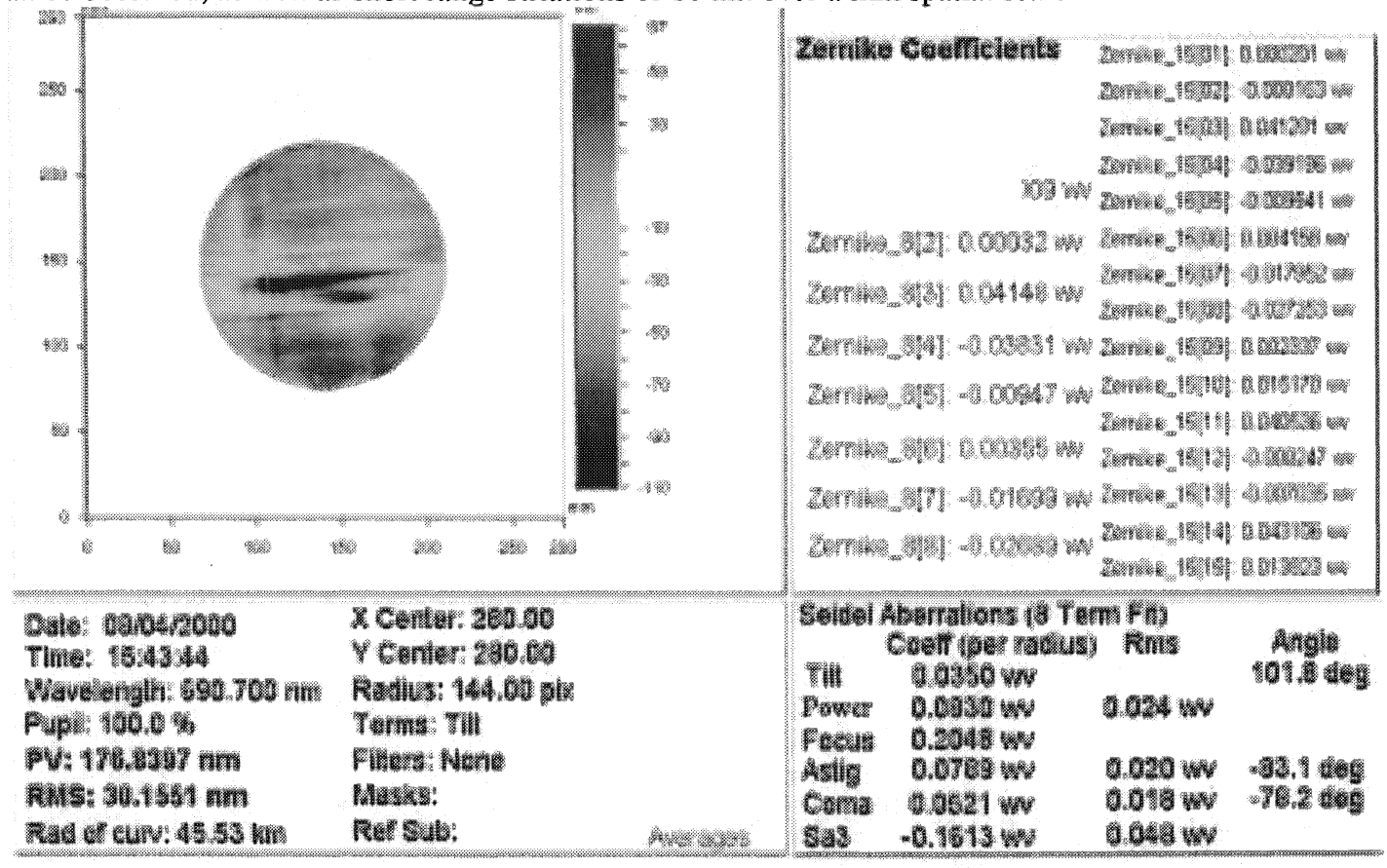

Figure 6: Optical inhomogeneity of sapphire substrate

To bring the optical homogeneity to the level of the requirements of table 3, we are pursuing a number of possible approaches. One possibility is computer controlled local polishing (also known as "spot polishing), where local inhomogeneities are measured and then compensated with local surface removal. Another possibility is the application of local coating, where the substrate in a coating chamber is moved behind a mask during the coating process.

\subsubsection{Absorption}

Absorption is a particular concern in the optics of the advanced LIGO interferometer because of the problem of thermal distortion from the high stored power $(800 \mathrm{~kW})$. We have initiated a study of absorption as a function of sapphire growth material, boule location, and annealing conditions. The absorption was measured with the following experimental arrangement (figure 7). A $1.06 \mu \mathrm{m}$ pump laser, intensity modulated with a chopping wheel, is used to heat the sapphire sample under test. The pump laser spot size is focused to $\sim 10 \mu \mathrm{m}$. A probe laser of spot size $1 \mathrm{~mm}$ is then directed through the sample, crossing the pump laser beam at an angle of $\sim 10$ degree. The sample heating causes a "dimple" to appear in the optical path of the probe, which, when projected by the imaging lens to the photodetector, gives rise to interference. The modulated intensity of the photodetected signal is then converted to a dc signal by the lock-in amplifier, which is proportional to the sample absorption. Translation of the test sample allows the spatial dependence of the absorp- 
tion to be extracted. Not shown in the figure is a laser of wavelength $514 \mathrm{~nm}$, which causes the fluorescence of $\mathrm{Ti}^{3+}, \mathrm{a}$ common sapphire contaminant.

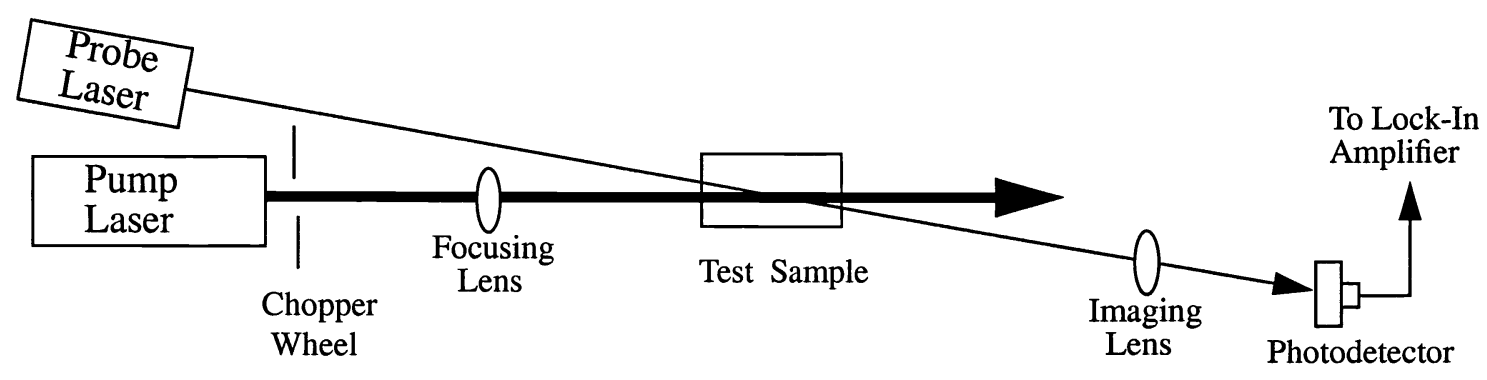

Figure 7: Absorption measurement setup

The nominal absorption of an as-grown samples of sapphire obtained from Crystal Systems was found to be $80+/$ $20 \mathrm{ppm} / \mathrm{cm}$. The experimental effort to lower the absorption to the level of $\sim 10 \mathrm{ppm} / \mathrm{cm}$ involved two separate paths: first, an attempt to identify starting materials and growth techniques that could result in the lowest level of impurity concentration, and second, annealing procedures that could provide the optimal impurity reduction state and/or heal intrinsic substrate defects (eg, oxygen vacancies.)

Identification of high purity sapphire was attempted by using a number of different sapphire starting growth materials, and also examining samples taken from varying boule locations to test for the possibility of impurity segregation. Measurement of $1064 \mathrm{~nm}$ absorption and Ti fluorescence of these samples showed no correlation, indicating that Ti impurities were not solely responsible for the absorption. The absorption also did not depend in any measurable way on starting material or boule location.

Annealing studies have suggested some possibilities for reducing the absorption. A high temperature air bake of 1 $\mathrm{cm}$ cube sapphire samples appears to reduce the bulk absorption, as shown in figure 8 .

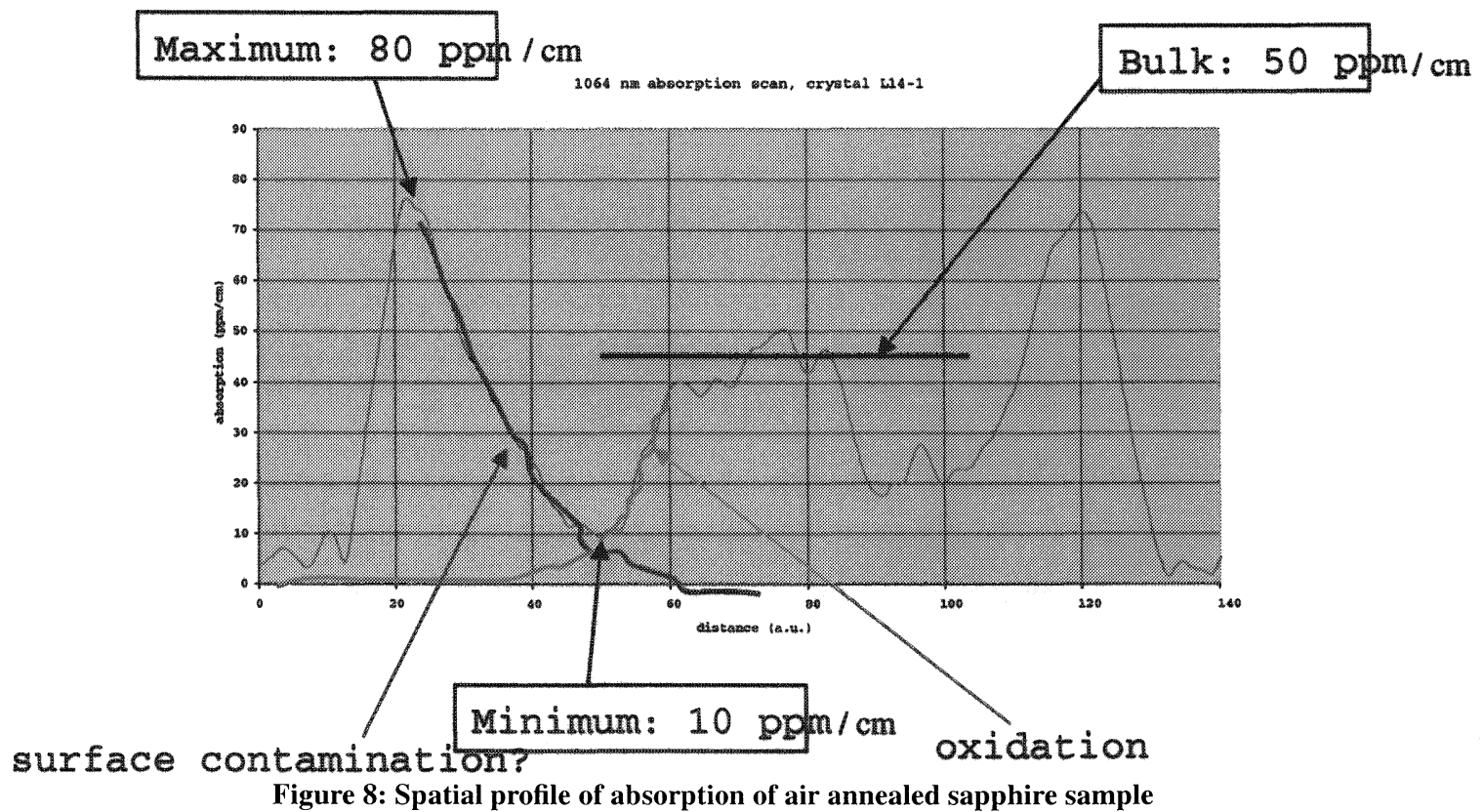


The figure shows a characteristic observed in many of the annealed samples, the presence of high absorption surface "wings". These wings, coupled with the observed dip of $10 \mathrm{ppm} / \mathrm{cm}$, are suggestive of two annealing diffusion waves: one which proceeds from the bulk outwards, reducing the nominal bulk absorption of $50 \mathrm{ppm} / \mathrm{cm}$, possibly through oxidation; and a contamination wave which proceeds from the surface inwards and which may be increasing the final sample absorption to the level at the wings. The minimum level of $10 \mathrm{ppm} / \mathrm{cm}$ seen in the figure encourages proceeding with the annealing studies. The next steps are to obtain new, clean annealing furnaces to attempt annealing at increased temperatures but without surface contamination effects. At that point it may be possible to revisit the question of the correlation of absorption with starting material.

\subsubsection{Birefringence}

A concern in the use of sapphire is the anisotropic property of the crystal. The anisotropy in the thermal expansion coefficient in the surface of the $\mathrm{m}$-axis substrate will cause differential expansion when heated by the coating process. When the substrate cools the ensuing uneven contraction will stress the coating, leading to birefringence.

Birefringence in the test mass coatings will affect the advanced LIGO in the following way. We assume that the polarization of the light incident on an arm cavity is at an angle $\alpha$ with respect to the optical axes of the coatings of both cavity optics. Then the light will experience a rotation of its polarization on reflection from the arm cavity of

$$
\Delta \phi=2 G_{a r m} \theta \sin \alpha \cos \alpha
$$

where $G_{a r m}$ is the optical gain of the arm cavity and $\theta$ is the coating birefringence (assumed the same for both cavity optics). This results in a fraction of power in the polarization orthogonal to the input polarization equal to $(\Delta \phi)^{2}$, which will be lost at the beamsplitter. Given a recycling gain of $G_{r c}$ which we wish to preserve, the power in the orthogonal direction should be no more than of order $0.1 / \mathrm{G}_{\mathrm{rc}}$. For small a value of $\alpha$ this leads to the condition ${ }^{8}$ :

$$
\frac{0.1}{G_{r c}}>4\left(G_{a r m} \theta \alpha\right)^{2}
$$

For the current nominal values of $\mathrm{G}_{\mathrm{rc}} \sim 15$ and $\mathrm{G}_{\mathrm{arm}} \sim 800$ we obtain $(\theta \alpha)<5 \times 10^{-5} \mathrm{rad}^{2}$ as an upper limit for the allowed product of birefringence and alignment to the optical axis of the sapphire coating.

To address this concern we have investigated the birefringence of m-axis sapphire with a Fabry-Perot cavity consisting of a high reflectance coated sapphire optic and a coated fused silica optic. By monitoring the transmission of light as a function of its polarization, the birefringence may be determined. The relation between the transmitted power $I$ and the difference in resonant frequency between the polarization eigenmodes $\delta v$ is given by:

$$
\frac{I}{I_{0}}=\cos ^{2}\left(\Phi+\Phi_{0}\right)+\frac{\sin ^{2}\left(\Phi+\Phi_{0}\right)}{1+\left(\frac{\delta V}{\Delta v_{H W H M}}\right)^{2}}
$$

where $I_{0}$ is the incident power, $\Phi$ is the input polarization angle, $\Phi_{0}$ is the initial offset angle of the input polarization with respect to the coating optical axes, and $\Delta v_{\mathrm{HWHM}}$ is the half-width of the cavity transmitted power resonance. With a cavity of $\Delta v_{\mathrm{HWMH}} \sim 10 \mathrm{kHz}$, the intensity dips of figure 9 correspond to a birefringence of $2.5 \times 10^{-4} \mathrm{rad}$. Measurement of 8 
points on the coating gave an uncertainty in the direction of the birefringence of about 10 degree. Together these values satisfy the upper limit for advanced LIGO.

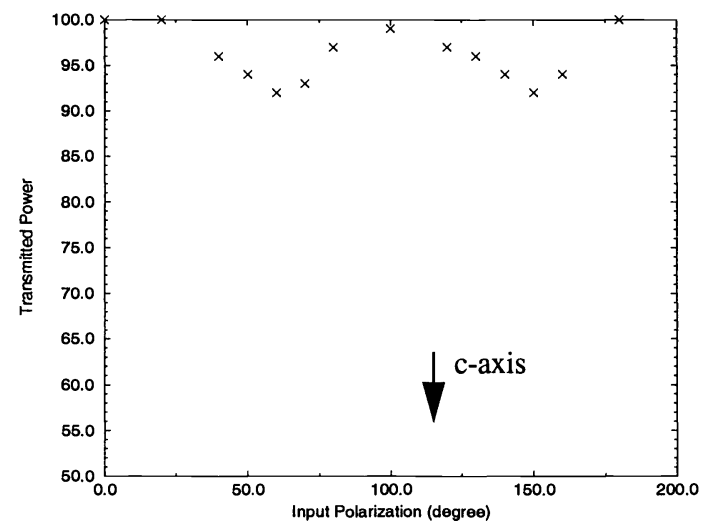

Figure 9: Measurement of birefringence of coated sapphire optic

\subsection{Coating development}

In the fabrication of optics for LIGO I, the performance of the low-loss, ion beam sputtered coatings provided by the vendor were sufficient. However, the coatings used were optimized for the needs of the ring-laser gyro industry which presents different requirements than those of gravitational wave interferometry. In particular, meeting the requirements of the advanced LIGO detector will necessitate an active development effort aimed at reducing absorption and mechanical loss in the coatings. We are now engaged in coating R\&D with two separate coating vendors, MLD Technologies, Mountain View, Ca., and SMA/Lyon, Lyon, France.

\subsubsection{Mechanical loss}

The mechanical loss of the optics is a critical parameter in determining the interferometer thermal noise. Though relatively thin, the lossy coatings provide a source of excess loss which may adversely impact the optics thermal noise. The relation of the mechanical loss of a coated and uncoated optic is given by:

$$
\phi_{\text {coated }}=\phi_{\text {uncoated }}+\frac{E_{\text {coatingface }}}{E_{\text {bulk }}} \phi_{\text {coatingface }}+\frac{E_{\text {barrelcoating }}}{E_{\text {bulk }}} \phi_{\text {barrelcoating }}
$$

where $\phi$ is the mechanical loss, and $E$ is the stored energy in the bulk, coating face, or barrel (in the measured substrates some of the coating had spilled onto the barrel.) Preliminary measurements of coated optics have been carried out with a ringdown apparatus, which determines the mechanical $Q$ of the optic. This device places the substrate under test in a vacuum chamber and excites a number of mechanical modes with an electrostatic driver. The amplitude of a specific mode is monitored with an interferometer and the $1 / \mathrm{e}$ time of its decay is measured. The loss is then given by $\phi=2 / \omega \tau=1 / \mathrm{Q}$, where $\omega$ is the mode frequency and $\tau$ is the decay time. After obtaining the losses of 6 modes of a coated substrate, eqn. 6 can be solved by multiple linear regression to obtain $\phi$ of the substrate, coating, and barrel coating. The results for two types of fused silica substrates are shown in Table 4.

An alternative approach to measuring the coating mechanical loss has been pursued by measuring the ringdown of fused silica disks before and after the application of a coating. In this case we have

$$
\phi_{\text {coating }}=\frac{V}{S} \frac{1}{\mu d}\left(\frac{1}{Q_{\text {coated }}}-\frac{1}{Q_{\text {uncoated }}}\right)
$$


Table 4: Coating Mechanical Losses

\begin{tabular}{|c|c|c|c|}
\hline Material & $\phi_{\text {substrate }}$ & $\phi_{\text {coating }}$ & $\phi_{\text {barrelcoating }}$ \\
\hline Corning 7940 fused silica & $3.7+/-0.5 \times 10^{-8}$ & $6.4+/-0.6 \times 10^{-5}$ & $6.9+/-0.4 \times 10^{-5}$ \\
\hline Corning 7980 fused silica & $5.6+/-0.9 \times 10^{-8}$ & $6.3+/-1.6 \times 10^{-5}$ & $6.3+/-0.9 \times 10^{-5}$ \\
\hline
\end{tabular}

where $V$ and $S$ are the substrate volume and surface area, $d$ is the coating thickness, and $\mu$ is a factor which depends on the sample geometry and mode of excitation. The value of the coating loss for a $24 \mu \mathrm{m}$ thick coating is $9.3+/-0.3 \times 10^{-5}$, in good agreement with the measurements of table 4.

These values of coating mechanical loss, when coupled to the full sized LIGO substrates, may have an effect on the interferometer sensitivity at the tens of percent level. To better understand and minimize this effect, we have begun a careful study of the factors in the coating design which may impact the mechanical loss: material used, thickness, and coating/ substrate surface interaction. Substrate Q's will be measured before and after the different coating designs are applied to obtain the effect of these parameters on $\phi_{\text {coating }}$ directly, allowing the optimal coating design for the advanced LIGO optics.

\subsubsection{Absorption}

Coating absorption, coupled to the increased laser power of the advanced LIGO interferometer, is a source of heat input which will cause optical distortion in the wavefront transmitted through the input test masses. Table 4 shows the level of distortion from both the coating and the substrate absorption. These numbers are appropriately compared to the design sagitta of the optics, of order $40 \mathrm{~nm}$ across the $6 \mathrm{~cm}$ radius beam spot; the research goal is, if possible, to develop coatings and substrates with a total thermal distortion less than the sagitta. It can be seen from the table that the distortion from coatings applied to fused silica is very large, because of the relatively low thermal conductivity of fused silica compared to sapphire. Thus for fused silica to be considered as a possible core optic material for the advanced LIGO, the coating absorption would need to be brought down by an order of magnitude. However, even for sapphire, the overall optical distortion would be attenuated by a decrease in coating absorption by a factor of 2-3. For this reason we are pursuing an empirical study of the absorption of a number of different coating materials in parallel with the coating mechanical loss study.

Table 5: Thermal Distortion of ITM Transmitted Wavefront

\begin{tabular}{|c|c|c|}
\hline \multirow{2}{*}{ Source of Absorption } & \multicolumn{2}{|c|}{ Test Mass Optical Distortion } \\
\cline { 2 - 3 } & Sapphire & Fused Silica \\
\hline \hline $\begin{array}{c}\text { Substrate } \\
\text { (Sapphire: } 10 \mathrm{ppm} / \mathrm{cm}) \\
\text { (Fused Silica: } 1 \mathrm{ppm} / \mathrm{cm})\end{array}$ & $20 \mathrm{~nm}$ & $40 \mathrm{~nm}$ \\
\hline $\begin{array}{c}\text { Coating } \\
(0.5 \mathrm{ppm})\end{array}$ & $25 \mathrm{~nm}$ & $400 \mathrm{~nm}$ \\
\hline
\end{tabular}

A further possibility to reduce the ITM thermal distortion is external thermal compensation, where a heating ring or spatially scanned laser is used to deposit heat in the optic to compensate the non-uniform heating caused by the absorbed stored interferometer power. Thermal compensation will be necessary in the event that the sapphire or coating absorption cannot be reduced to managable levels. 


\section{SUMMARY}

At the present time we have completed the installation of the LIGO I optics. All optics have been tested at Caltech and meet specifications for surface figure, radius of curvature, scatter, and coating and substrate absorption. The sole deviation from specification has been found to be the beam splitter ROC, which was altered by the coating compressive strain. In-situ characterization of the total loss of the Hanford $2 \mathrm{~km}$ interferometer optics shows agreement with these specifications.

Development of sapphire optics for the advanced LIGO interferometer is underway. The sapphire characteristics of polishability and birefringence have been studied and found to satisfy the advanced LIGO requirements. Sapphire optical homogeneity and absorption are offering a research challenge: development of a polishing technique to compensate local homogeneity variations is continuing, as are efforts to develop contaminant-free furnaces where high-temperature annealing can be used to lower sapphire absorption. Finally, a study of the mechanisms of coating mechanical and absorption loss has been initiated to attempt to design coatings optimized for the advanced interferometer.

\section{ACKNOWLEDGMENTS}

This work is supported by the National Science Foundation under Cooperative Agreement PHY-9210038.

\section{REFERENCES}

1. A. Abramovici, et al., Science 256 (1992) 325-333

2. B. Bochner, thesis, Massachusetts Institute of Technology, 1998

3. W. Winkler, K. Danzmann, A. Rudiger, R. Schilling, "Heating by optical absorption and the performance of interferometric gravitational wave detectors", Phys Rev A44, 7022

4. D. McClelland, J. Camp, J. Mason, W. Kells, S. Whitcomb, "Arm cavity resonant sideband control for laser interferometric gravitational wave detectors," Optics Letters 24, 1014 (1999)

5. J. Camp , H.Armandula, G.Billingsley, M.Hrynevych, W.Kells, A. Lazzarini, D. Li, R. Weiss, S.Whitcomb, H.Yamamoto, "Requirements and testing of LIGO core optics", in Proceedings of the 2nd TAMA International Workshop on Graviational Wave Detction, S. Kawamura and N. Mio, eds. (Universal Academy Press, Inc., Tokyo, 2000), pp. 279-288

6. C. Walsh, A.Leistner, B. Oreb, et.al., "Manufacture of LIGO core optics at CSIRO," Proc. SPIE Vol. 3782, p. 214-223, Optical Manufacturing and Testing III, H. Philip Stahl; Ed. Nov. (1999)

7. B Oreb, A Leistner, G. Billingsley, B. Kells and J. Camp "Interferometric measurement of refractive index inhomogeneity on polished sapphire substrates: application to LIGO-II," Proc. SPIE Vol. 4451 Optical Manufacturing and Testing IV, H. Philip Stahl: Ed. Aug. (2001)

8. J. Camp, W. Kells, E. Gustafson, M. Fejer, "Measurement of birefringence of low-loss high reflectance coating of maxis sapphire", Applied Optics 40, 3753 (2001) 


\section{LIGO optics: initial and advanced (4679-03)}

Jordan Camp, LIGO Project, California Institute of Technology, USA

Q. Do you know the identity of the impurities in the sapphire?

A. We had suspected titanium $3+$ but fluorescence measurements didn't correlate that very well with the absorption of our test samples so we know that at least there is something else going on, but we don't know what that is.

\section{Q. Did you look for chromium?}

A. We did some GDS measurements in the various samples we looked at but none of that was particularly revealing.

Q. If you just hit it with the blue-green laser, and if there is a tiny amount of chromium it will fluoresce at $693 \mathrm{~nm}$, very strongly. Try that. One second question, how much did that little earthquake in Washington affect your LIGO experiments?

A. A couple of months. Our suspended FP cavities are very susceptible to that; they get knocked out of alignment. It is a good earthquake detector.

Q. This is a follow on from what Greg said. You might look at 3+ transition metals. Iron tends to be a real problem and it gives a very broad band, kind of hard to detect. But you can fiddle with annealing. My question revolves around what sort of pressure levels do you need to maintain around the mirrors and then in the whole beam line?

A. There are a number of effects from pressure. One is you don't want the optics bouncing around from the shot noise, from the pressure hitting the optics. That imposes a pressure requirement of something like $10^{-5}$ torr or something like that. Another more serious problem is a variation of the index of refraction of the vacuum from gas molecules passing through the light beam. The most serious one for us depends upon the polarizability of the molecules floating through the beam. The most serious molecule for us is hydrogen. We have to hold to something like $10^{-9}$ torr. We had a huge research program to study this problem and we found that we needed to use specially annealed steel from the steel producing plants so that the steel wouldn't outgas. The out-gassing of $\mathrm{H}_{2}$ from the specialty steel was low enough. We did these studies when we were building the beam tube. It appears that in the vacuum system we got hydrogen down to about the $10^{-9}$ torr level. That's the real requirement.

Q. How many scatter detectors do you have in your scatterometer?

A. Six.

Q. Have you any estimation or measurements on the relaxation of the coating stress over time that might modify the change in the focal length of the system? 
A. No, do you know something about that?

A. Well there have been studies on that over time; there has been a real relaxation.

Q. Can you give me a reference on that?

A. Yes with a little searching. 